\title{
A shape sensitivity analysis approach based on the boundary element method
}

\author{
T. Matsumoto ${ }^{1}$, T. Takahashi ${ }^{1}$, K. Shibata ${ }^{2} \&$ T. Yamada ${ }^{1}$ \\ ${ }^{1}$ Nagoya University, Japan \\ ${ }^{2}$ Kyushu Electric Power Co. Inc., Japan
}

\begin{abstract}
A revised shape design sensitivity formulation is presented for elastostatic problems based on the adjoint method and the boundary element method. The objective function is assumed as a functional consisting of the boundary quantities and those given at some finite number of points in the domain of the solid. The gradient of the objective function is derived and an adjoint state is introduced so that the unknown sensitivity coefficients of the displacement and traction on the boundary and in the domain are eliminated from the gradient expression. Since the original boundary value problem and the adjoint problem are governed by the same differential equations and the boundary condition types, and also the derived sensitivity formulation is expressed with only the boundary integrals and the quantities at some discrete points in the domain, the boundary element method can be used as the effective computational tool. Also, the recent development of the fast-multipole boundary element method enables a large-scale shape optimization analysis of complicated structures. The validity of the derived formulation is tested through some numerical example problems.

Keywords: elastostatics, shape sensitivity, adjoint method, boundary element method, topology optimization.
\end{abstract}

\section{Introduction}

Although the boundary element method (BEM) has an advantage of boundary only modeling, it has not become a popular simulation tool in engineering applications because it required high storage and computation costs. For shape and topology optimization problems, the finite element method (FEM) has widely been used as the computational method. However, following the development of 
fast computation algorithm $[1,2]$ in recent decades, BEM may become a powerful alternative to FEM in shape optimization problems.

Shape optimization as well as topology optimizations problems define objective functions of the quantities defined on the boundary and in the domain. All such quantities are also functions of shape parameters, and the optimum values of the shape parameters minimizing the objective functions are calculated. The gradients, or sensitivities, of the objective function with respect to the design variables are also needed in most of the solution procedures to find the direction to the minimum point of the objective function. The gradient is related to a variation of the objective function, and consists of the sensitivities of the boundary and internal quantities. To calculate these sensitivities, direct differentiation method [3-5] and adjoint variable method [6,7] has been proposed. When using BEM, the direct differentiation method uses an additional boundary integral equation obtained by differentiating the original boundary integral equation with respect to an arbitrary shape design variable. The direct differentiation is, however, costly for problems with a large number of design variables because the additional boundary integral equation for the sensitivity must be solved for every design variable. The adjoint variable method defines an additional system that eliminates the unknown sensitivities on the boundary and in the domain. Therefore this method is more efficient because we have to repeat the boundary element calculation only for the original problem and the adjoint problem to calculate the gradient of the objective function.

In this paper, we consider an objective function that is appropriate to evaluate by means of BEM. The objective function is assumed to consist only of the quantities on the boundary and at some discrete points in the domain. Adjoint variable method is applied to the defined objective function and some numerical examples are shown to demonstrate the effectiveness of the approach.

\section{Formulations}

\subsection{Boundary element method for elastostatics}

The governing differential equation for linear isotropic elastic solids is the following Navier's equation:

$$
C_{i j k l} u_{k, l i}+b_{j}=G u_{j, k k}+\frac{G}{(1-2 \nu)} u_{k, k j}+b_{j}=0 \text { in } \Omega
$$

where $C_{i j k l}$ denotes the elastic tensor, $u_{i}$ and $b_{i}$ are the displacement and body force vectors, respectively, $G$ is the shear modulus, $\nu$ is Poisson's ratio, and $\Omega$ is the domain under consideration. The index is assumed to change from 1 to 3 for three-dimensional problems, and from 1 to 2 for two-dimensional case. For terms with repeated indices, summation convention is assumed. The indices after a comma denote differentiations with respect to the coordinate axes. 
The boundary conditions are as follows:

$$
\begin{aligned}
& u_{i}=\bar{u}_{i} \text { on } \Gamma_{u} \\
& t_{i}=\bar{t}_{i} \quad \text { on } \Gamma_{t}
\end{aligned}
$$

where $t_{i}$ is traction, $\bar{u}_{i}$ and $\bar{t}_{i}$ are the prescribed known functions of $u_{i}$ and $t_{i}$ on some parts of the boundary $\Gamma_{u}$ and $\Gamma_{t}$, respectively.

The boundary integral equation derived corresponding to Eq. (1) becomes [8]

$$
\begin{aligned}
c_{i j} u_{i}(y) & +\int_{\Gamma} t_{i j}^{*}(x, y) u_{i}(x) d \Gamma(x)=\int_{\Gamma} u_{i j}^{*}(x, y) t_{i}(x) d \Gamma(x) \\
& +\int_{\Omega} u_{i j}^{*}(x, y) b_{i}(x) d \Omega(x), \quad y \in \Gamma
\end{aligned}
$$

where $x$ and $y$ are points on the boundary, $c_{i j}$ is a constant tensor, becoming $1 / 2 \delta_{i j}$ when $y$ lies at a smooth part of the boundary, $u_{i j}^{*}$ is the fundamental solution, and $t_{i j}^{*}$ is the traction related to $u_{i j}^{*}$. For two-dimensional, plane strain, case, $u_{i j}^{*}$ and $t_{i j}^{*}$ are given as

$$
\begin{gathered}
u_{i j}^{*}(x, y)=\frac{1}{8 \pi G(1-\nu)}\left\{(3-4 \nu) \delta_{i j} \ln \left(\frac{1}{r}\right)+r_{, i} r_{, j}\right\} \\
t_{i j}^{*}(x, y)=\frac{-1}{4 \pi(1-\nu) r}\left[\frac{\partial r}{\partial n}\left\{(1-2 \nu) \delta_{i j}+2 r_{, i} r_{, j}\right\}\right. \\
\left.+(1-2 \nu)\left(r_{, i} n_{j}-r_{, j} n_{i}\right)\right]
\end{gathered}
$$

where $r$ is the distance between $x$ and $y, n_{i}$ is the unit outward normal vector at $x$, and $\partial r / \partial n$ is the derivative of $r$ in $n_{i}$ direction at $x$.

In what follows, we assume that no body force exists in the domain for simplicity. Discretizing Eq. (4), we have the following system of algebraic equations:

$$
[H]\{u\}=[G]\{t\}
$$

and rearranging this so that all the unknowns come to the left-hand side and all the others to the right-hand side result in

$$
[A]\{X\}=\{Y\}
$$

where $\{X\}$ is the vector consisting only of unknown nodal values, while $\{Y\}$ is the vector obtained by multiplying the known nodal values with corresponding parts of the coefficient matrix.

Once Eq. (8) is solved we obtain all the displacement and tractions on the boundary. The stress component on the boundary can be calculated using them 
by

$$
\begin{aligned}
\sigma_{i j}= & \frac{1}{1-\nu}\left(\nu \delta_{i j}-n_{i} n_{j}\right) t_{k} n_{k}+t_{i} n_{j}+t_{j} n_{i} \\
& +G\left\{\frac{2 \nu}{1-\nu}\left(2 \delta_{i j}-n_{i} n_{j}\right) \alpha_{k}+\left(\delta_{i k}-n_{i} n_{k}\right) \alpha_{j}+\left(\delta_{j k}-n_{j} n_{k}\right) \alpha_{i}\right\} \frac{\partial u_{k}}{\partial \alpha} \\
& +G\left\{\frac{2 \nu}{1-\nu}\left(2 \delta_{i j}-n_{i} n_{j}\right) \beta_{k}+\left(\delta_{i k}-n_{i} n_{k}\right) \beta_{j}+\left(\delta_{j k}-n_{j} n_{k}\right) \beta_{i}\right\} \frac{\partial u_{k}}{\partial \beta}
\end{aligned}
$$

where $\alpha_{k}$ and $\beta_{k}$ are tangential vectors on the boundary, and $\partial u_{k} / \partial \alpha$ and $\partial u_{k} / \partial \beta$ are corresponding tangential derivatives of $u_{k}$.

The internal displacement can be calculated by using Eq. (4) with $c_{i j}=\delta_{i j}$. The formula for calculating internal stresses is also obtained by differentiating Eq. (4) with $c_{i j}=\delta_{i j}$ at an internal point and substituting it into Hooke's law [8].

\subsection{Objective function of shape optimization problem and its sensitivity}

We consider the following objective function

$$
J=\int_{\Gamma} g\left(u_{i}, t_{i}\right) d \Gamma(x)+\sum_{s} \int_{\Omega} h\left(u_{i}, \sigma_{i j}\right) \delta\left(x-z^{s}\right) d \Omega, \quad z^{s} \in \Omega(x)
$$

where $\Gamma$ denotes the boundary, $\Omega$ the domain, $u_{i}$ and $t_{i}$ the displacement and traction, respectively, $\sigma_{i j}$ the stress components, $z_{s},(s=1,2, \ldots)$ the discrete points in the domain, and $\delta\left(x-z_{s}\right)$ the Dirac delta function. $g\left(u_{i}, t_{i}\right)$ is a function defined with $u_{i}$ and $t_{i}$ on the boundary, while $h\left(u_{i}, \sigma_{i j}\right)$ is that defined in the domain.

Note that the second domain integral is not in fact an integral because Dirac's delta functions exist in the integrands, therefore, this type of objective function is quite appropriate to treat with BEM.

The gradient of $J$ with respect to an arbitrary shape design variable becomes

$$
\begin{aligned}
J^{\prime}= & \int_{\Gamma}\left(\frac{\partial g}{\partial u_{i}} \dot{u}_{i}+\frac{\partial g}{\partial t_{i}} \dot{t}_{i}\right) d \Gamma+\int_{\Gamma} g d \Gamma \\
& +\sum_{s} \int_{\Omega}\left(\frac{\partial h}{\partial u_{i}} \dot{u}_{i}+\frac{\partial h}{\partial \sigma_{i j}} \dot{\sigma}_{i j}\right) \delta\left(x-z^{s}\right) d \Omega \\
& +\sum_{s} \int_{\Omega} h\left(u_{i}, \sigma_{i j}\right) \delta\left(x-z^{s}\right) d \dot{\Omega}+\sum_{s} \int_{\Omega} h\left(\left(u_{i}, \sigma_{i j}\right) \dot{\delta}\left(x-z^{s}\right) d \Omega\right.
\end{aligned}
$$


An overscribed dot $\left(^{*}\right)$ in the above expression denotes a material derivative [9], $\dot{u}_{i}, \dot{t}_{i}, \dot{\sigma}_{i j}$ are understood in the following sense:

$$
\begin{gathered}
\dot{u}_{i}=u_{i}^{\prime}+u_{i, j} \dot{x}_{j} \\
\dot{t}_{i}=t_{i}^{\prime}+t_{i, j} \dot{x}_{j} \\
\dot{\sigma}_{i j}=\sigma_{i j}^{\prime}+\sigma_{i j, k} \dot{x}_{k}
\end{gathered}
$$

where a prime $\left(^{\prime}\right)$ denotes a differentiation with respect to the design variable before the shape change is applied. Also, $d \Gamma$ and $\dot{d} \Omega$ are written as follows [9]:

$$
\begin{aligned}
\dot{d} \Gamma & =\left(\dot{x}_{m, m}-\dot{x}_{i, j} n_{i} n_{j}\right) d \Gamma \\
\dot{d} \Omega & =\dot{x}_{m, m} d \Omega
\end{aligned}
$$

We find from Eq. (11) that in order to evaluate Eq. (10) we have to calculate the sensitivities of the $\dot{u}_{i}, \dot{t}_{i}, \dot{\sigma}_{i j}$ as many as the number of the design variable. Although these quantities can be calculated by using the direct differentiation method based on the differentiation of the boundary integral equation with respect to the design variable, it is not efficient when the number of the design variables is large. Therefore, we apply the adjoint variable method to eliminate these unknown sensitivities from Eq. (11). We now observe

$$
\left(\dot{u_{k, l}}\right)=\left(\dot{u}_{k}\right)_{, l}-u_{k, m} \dot{x}_{m, l}
$$

where $(\dot{)})$ denotes a material derivative of the quantity enclosed with parentheses. Using Eq. (17) we have the material derivative of the stress components as follows:

$$
\begin{aligned}
\dot{\sigma}_{i j} & =C_{i j k l}\left(\dot{u_{k, l}}\right) \\
& =C_{i j k l}\left(\dot{u}_{k}\right)_{, l}-C_{i j k l} u_{k, m} \dot{x}_{m, l}
\end{aligned}
$$

Using Eq. (18), we can modify the integral of $\dot{\sigma}_{i j}$ in Eq. (11) as

$$
\begin{gathered}
\int_{\Omega} \frac{\partial h}{\partial \sigma_{i j}} \dot{\sigma}_{i j} \delta\left(x-z^{s}\right) d \Omega=\int_{\Omega} \frac{\partial h}{\partial \sigma_{i j}} C_{i j k l}\left(\dot{u}_{k}\right)_{, l} \delta\left(x-z^{s}\right) d \Omega \\
-\int_{\Omega} \frac{\partial h}{\partial \sigma_{i j}} C_{i j k l} u_{k, m} \dot{x}_{m, l} \delta\left(x-z^{s}\right) d \Omega \\
=-\int_{\Omega}\left[\frac{\partial h}{\partial \sigma_{i j}} \delta\left(x-z^{s}\right)\right]_{, l} C_{i j k l} \dot{u}_{k} d \Omega \\
-\int_{\Omega} \frac{\partial h}{\partial \sigma_{i j}} C_{i j k l} u_{k, m} \dot{x}_{m, l} \delta\left(x-z^{s}\right) d \Omega
\end{gathered}
$$


Then, Eq. (11) becomes

$$
\begin{aligned}
J^{\prime}= & \int_{\Gamma}\left(\frac{\partial g}{\partial u_{i}} \dot{u}_{i}+\frac{\partial g}{\partial t_{i}} \dot{t}_{i}\right) d \Gamma+\int_{\Gamma} g d \Gamma+\sum_{s} \int_{\Omega} \frac{\partial h}{\partial u_{i}} \dot{u}_{i} \delta\left(x-z^{s}\right) d \Omega \\
& -\sum_{s} \int_{\Omega}\left(\frac{\partial h}{\partial \sigma_{i j}}\right)_{, l} C_{i j k l} \dot{u}_{k} \delta\left(x-z^{s}\right) d \Omega \\
& -\sum_{s} \int_{\Omega} \frac{\partial h}{\partial \sigma_{i j}} C_{i j k l} \dot{u}_{k} \delta_{, l}\left(x-z^{s}\right) d \Omega+\sum_{s} \int_{\Omega} h \dot{\delta}\left(x-z^{s}\right) d \Omega \\
& -\sum_{s} \int_{\Omega} \frac{\partial h}{\partial \sigma_{i j}} C_{i j k l} u_{k, m} \dot{x}_{m, l} \delta\left(x-z^{s}\right) d \Omega+\sum_{s} \int_{\Omega} h \delta\left(x-z^{s}\right) d \Omega
\end{aligned}
$$

We consider the following augmented objective function instead of Eq. (10):

$$
P=J+I
$$

where $I$ is the integral of the left-hand side of Navier's equation times Lagrange multipliers $\lambda_{j},(j=1,2,3)$ over the domain, as follows:

$$
I=\int_{\Omega} \lambda_{j} C_{i j k l} u_{k, l i} d \Omega
$$

where $C_{i j k l}$ is the elastic constant tensor. Integrating $I$ by parts gives the following weak form:

$$
I=\int_{\Gamma} \lambda_{j} t_{j} d \Gamma-\int_{\Omega} \lambda_{j, i} C_{i j k l} u_{k, l} d \Omega
$$

From Eq. (23), we have

$$
\begin{aligned}
I^{\prime}= & \int_{\Gamma} \dot{\lambda}_{j} t_{j} d \Gamma+\int_{\Gamma} \lambda_{j} \dot{t}_{j} d \Gamma+\int_{\Gamma} \lambda_{j} t_{j} \dot{d \Gamma} \\
& -\int_{\Omega}\left(\dot{\lambda_{j, i}}\right) C_{i j k l} u_{k, l} d \Omega-\int_{\Omega} \lambda_{j, i} C_{i j k l}\left(\dot{u_{k, l}}\right) d \Omega-\int_{\Omega} \lambda_{j, i} C_{i j k l} u_{k, l} \dot{d} \dot{\Omega}
\end{aligned}
$$

Again, we observe some relationships for the material derivative of the Lagrange multiplier as

$$
\begin{aligned}
\dot{\lambda}_{j} & =\lambda_{j, m} \dot{x}_{m} \\
\left(\dot{\lambda_{j, i}}\right) & =\lambda_{j, i m} \dot{x}_{m}
\end{aligned}
$$


Using Eqs. (17), (25) and (26) into Eq. (24) and applying integration by parts give

$$
\begin{aligned}
I^{\prime}= & \int_{\Gamma} \lambda_{j, m} \dot{x}_{m} t_{j} d \Gamma+\int_{\Gamma} \lambda_{j} \dot{t}_{j} d \Gamma+\int_{\Gamma} \lambda_{j} t_{j} d \Gamma \\
& -\int_{\Gamma} \lambda_{j, i} C_{i j k l} \dot{u}_{k} n_{l} d \Gamma+\int_{\Gamma} \lambda_{j, i} C_{i j k l} u_{k, m} \dot{x}_{m} n_{l} d \Gamma \\
& +\int_{\Omega} \lambda_{j, i l} C_{i j k l} \dot{u}_{k} d \Omega-\int_{\Omega} \lambda_{j, i l} C_{i j k l} u_{k, m} \dot{x}_{m} d \Omega \\
& -\int_{\Gamma} \lambda_{j, i} C_{i j k l} u_{k, l} \dot{x}_{m} n_{m} d \Gamma
\end{aligned}
$$

Thus, we obtain the gradient of the augmented objective function $P$, as follows:

$$
\begin{aligned}
& P^{\prime}=\int_{\Gamma_{t}}\left(\frac{\partial g}{\partial u_{k}}-\tau_{k}\right) \dot{u}_{k} d \Gamma+\int_{\Gamma_{u}}\left(\frac{\partial g}{\partial u_{k}}-\tau_{k}\right) \dot{u}_{k} d \Gamma \\
& +\int_{\Gamma_{t}}\left(\frac{\partial g}{\partial t_{i}}+\lambda_{i}\right) \dot{t}_{i} d \Gamma+\int_{\Gamma_{u}}\left(\frac{\partial g}{\partial t_{i}}+\lambda_{i}\right) \dot{t}_{i} d \Gamma \\
& +\int_{\Omega}\left\{C_{i j k l} \lambda_{j, i l}+\sum_{s}\left[\frac{\partial h}{\partial u_{k}} \delta\left(x-z^{s}\right)-\left(\frac{\partial h}{\partial \sigma_{i j}}\right)_{, l} C_{i j k l} \delta\left(x-z^{s}\right)\right.\right. \\
& \left.\left.-\frac{\partial h}{\partial \sigma_{i j}} C_{i j k l} \delta_{, l}\left(x-z^{s}\right)\right]\right\} \dot{u}_{k} d \Omega \\
& +\int_{\Gamma} g \dot{d} \Gamma+\int_{\Gamma} \lambda_{j, m} \dot{x}_{m} t_{j} d \Gamma \\
& +\int_{\Gamma} \lambda_{j} t_{j} \dot{d} \Gamma+\int_{\Gamma} \tau_{k} u_{k, m} \dot{x}_{m} d \Gamma \\
& -\int_{\Gamma} \lambda_{j, i} C_{i j k l} u_{k, l} \dot{x}_{m} n_{m} d \Gamma-\int_{\Omega} \lambda_{j, i l} C_{i j k l} u_{k, m} \dot{x}_{m} d \Omega \\
& +\sum_{s} \int_{\Omega} h \dot{\delta}\left(x-z^{s}\right) d \Omega \\
& -\sum_{s} \int_{\Omega} \frac{\partial h}{\partial \sigma_{i j}} C_{i j k l} u_{k, m} \dot{x}_{m, l} \delta\left(x-z^{s}\right) d \Omega \\
& +\sum_{s} \int_{\Omega} h \delta\left(x-z^{s}\right) \dot{d} \Omega
\end{aligned}
$$

where $\tau_{k}$ is defined as the traction corresponding to $\lambda_{j}$ as

$$
\tau_{k}=C_{i j k l} \lambda_{j, i} n_{l}
$$


Notice that $\dot{u}_{i}$ and $\dot{t}_{i}$ are also known on $\Gamma_{u}$ and $\Gamma_{t}$, respectively, whereas $\dot{u}_{i}$ on $\Gamma_{t}, \dot{u}_{i}$ on $\Gamma_{u}$, and $\dot{u}_{i}$ in $\Omega$ are all unknown. Therefore, in order to eliminate these unknown sensitivities from Eq. (28), we consider the following adjoint problem for $\lambda_{i}$ :

$$
\begin{gathered}
C_{i j k l} \lambda_{j, i l}(x)+\sum_{s}\left\{\frac{\partial h}{\partial u_{k}} \delta\left(x-z^{s}\right)-\left(\frac{\partial h}{\partial \sigma_{i j}}\right)_{, l} C_{i j k l} \delta\left(x-z^{s}\right)\right. \\
\left.-\frac{\partial h}{\partial \sigma_{i j}} C_{i j k l} \delta_{, l}\left(x-z^{s}\right)\right\}=0, \quad x \in \Omega \\
\tau_{k}(x)=\frac{\partial g}{\partial u_{k}}(x) \quad x \in \Gamma_{t} \\
\lambda_{i}(x)=-\frac{\partial g}{\partial t_{i}}(x) \quad x \in \Gamma_{u}
\end{gathered}
$$

Equation (30) is the same as Navier's equation with body force terms at discrete points. The boundary conditions given by Eqs. (31) and (32) have the same type of the original boundary condition given by Eqs. (2) and (3). Therefore, we can solve the adjoint problem very efficiently using the same coefficient matrices of BEM.

By using $\lambda_{i}$ as the solution of Eqs. (30), (31), and (32), the gradient of $P$ can be calculated by using the following expression.

$$
\begin{aligned}
P^{\prime}= & \int_{\Gamma_{u}}\left(\frac{\partial g}{\partial u_{i}}-\tau_{i}\right) \dot{u}_{i} d \Gamma+\int_{\Gamma_{t}}\left(\frac{\partial g}{\partial t_{i}}+\lambda_{i}\right) \dot{t}_{i} d \Gamma \\
& +\int_{\Gamma} g \dot{d} \Gamma+\int_{\Gamma} \lambda_{j, m} \dot{x}_{m} t_{j} d \Gamma+\int_{\Gamma} \lambda_{j} t_{j} d \Gamma \\
& +\int_{\Gamma} \tau_{k} u_{k, m} \dot{x}_{m} d \Gamma-\int_{\Gamma} \lambda_{i, j} \sigma_{i j} \dot{x}_{m} n_{m} d \Gamma \\
& -\sum_{s}\left\{\frac{\partial h}{\partial \sigma_{i j}} \sigma_{i j, m} \dot{x}_{m}+\frac{\partial h}{\partial u_{i}} u_{i, m} \dot{x}_{m}+h \dot{x}_{m, m}\right\}
\end{aligned}
$$

where $u_{i, j}$ can be calculated by using

$$
u_{i, j}=\frac{\partial u_{i}}{\partial n} n_{j}+\frac{\partial u_{i}}{\partial \alpha} \alpha_{j}+\frac{\partial u_{i}}{\partial \beta} \beta_{j}
$$

with

$$
\begin{aligned}
\frac{\partial u_{i}}{\partial n}= & \frac{1}{G}\left(t_{i}-\frac{1}{2(1-\nu)} t_{k} n_{k} n_{i}\right)-\left(\frac{\nu}{1-\nu} n_{i} \alpha_{k}+n_{k} \alpha_{i}\right) \frac{\partial u_{k}}{\partial \alpha} \\
& -\left(\frac{\nu}{1-\nu} n_{i} \beta_{k}+n_{k} \beta_{i}\right) \frac{\partial u_{k}}{\partial \beta}
\end{aligned}
$$

The formula to calculate $\lambda_{i, j}$ is obtained by simply replacing $u$ in Eqs. (34) and (35) with $\lambda$. 


\section{Numerical examples}

We consider a rectangular plate, subjected to a roller support on one end and a uniform tensile stress $\bar{t}=200$ [MPa] on the other end, as shown in Figure 1. Young's modulus and Poisson's ratio are assumed as $216 \mathrm{GPa}$ and 0.3 , respectively. The size of the plate is initially set as $L_{1}=0.1[\mathrm{~m}]$ and $L_{2}=0.02[\mathrm{~m}]$.

The functions $g$ and $h$ in Eq. (10) are given as follows:

$$
\begin{gathered}
g=0 \\
h=\frac{1}{2}\left(u_{1}(\mathrm{P})-0.00004\right)^{2}
\end{gathered}
$$

In this example, we observe that changing $L_{1}$ yields a different value of $u_{1}$, thus, $L_{1}$ is chosen to be a design variable to validate the derived formulas.

The boundaries of the rectangular plate is discretized into quadratic elements uniformly. We show in Table 1 the sensitivities of the objective function and errors obtained for different number of elements using the present approach.

Next we consider another rectangular plate model, as shown in Figure 2. Both sides of the plate are fixed and a concentrated force $F=2.67 \times 10^{6}[\mathrm{~N}]$ is applied at the center of the lower boundary. We intend to change the von Mises stress at internal point $\mathrm{P}$ to the target value $\bar{\sigma}=30[\mathrm{MPa}]$ by changing the $x_{2}$ coordinate of the nodes $\mathbf{a}, \mathbf{b}$, and $\mathbf{c}$ on a part of the boundary $\Gamma_{D}$. The related functions of the

Table 1: Sensitivities and their errors obtained for various number of element discretization of the rectangular plate model.

\begin{tabular}{ccc}
\hline Number of nodes & Sensitivity $P^{\prime}$ & Error [\%] \\
\hline \hline 24 & $-4.9434 \times 10^{-9}$ & $3.37 \times 10^{-1}$ \\
48 & $-4.9397 \times 10^{-9}$ & $4.49 \times 10^{-1}$ \\
120 & $-4.9622 \times 10^{-9}$ & $5.02 \times 10^{-3}$ \\
240 & $-4.9619 \times 10^{-9}$ & $2.82 \times 10^{-4}$ \\
\hline
\end{tabular}

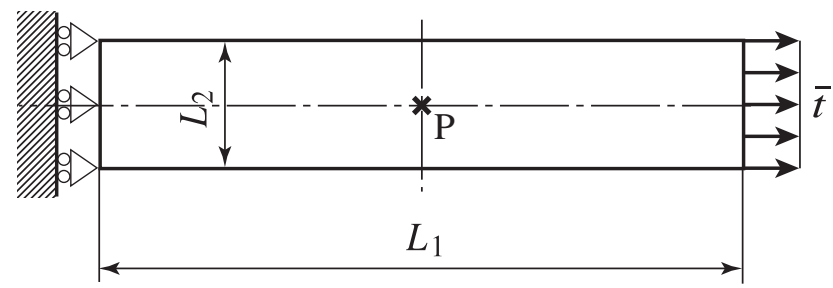

Figure 1: A rectangular plate subjected to uniform tensile stress. 


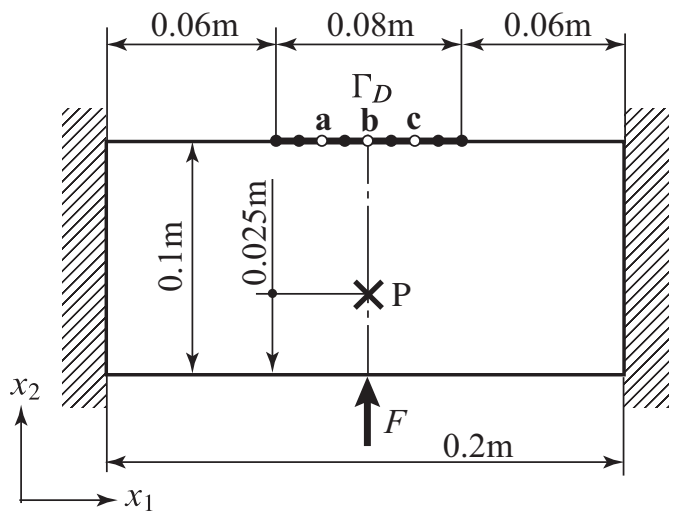

Figure 2: A rectangular plate subjected to a concentrated force.

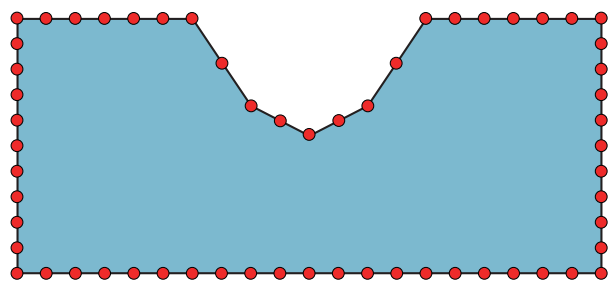

Figure 3: Final shape of the rectangular plate subjected to a concentrated force.

objective function are given as

$$
\begin{gathered}
g=0 \\
h=\frac{1}{2}\left(\sigma_{\mathrm{M}}(P)-\bar{\sigma}\right)^{2}
\end{gathered}
$$

where $\sigma_{\mathrm{M}}(P)$ is the von Mises stress at the internal point $\mathrm{P}$. The entire boundary is discretized into 30 quadratic elements uniformly. The concentrated force is given as an equivalent traction at the node placed at the point where the concentrated force is acting. In Figure 3 is shown the final shape of the plate obtained by the present procedure for calculating the shape sensitivities. The von Mises stress at point $\mathrm{P}$ in the optimum shape is found to be $3.0034128 \times 10^{7} \mathrm{~Pa}$, which is turned out to be very close to the target stress value.

\section{Concluding remarks}

A shape sensitivity expression for objective functions appropriate to evaluate using BEM, has been derived based on the adjoint variable method. The objective function consists of only boundary integrals of the displacement and traction, and values defined with the internal displacement and stress at discrete points in the 
domain. The differential equation of the adjoint system has become a Navier's equation with body forces at the discrete points in the domain, and the boundary conditions are of the same type as those of the original problem. Therefore, the same coefficient matrices can be consistently used also for the adjoint problem. The derived adjoint variable approach was applied to some numerical examples to validate its effectiveness.

\section{References}

[1] Rokhlin, V., Rapid solution of integral equations of classical potential theory. Journal of Computational Physics, 60(2), pp. 187-207, 1985.

[2] Yoshida, K., PhD Thesis, Kyoto University, Japan, 2001.

[3] Barone, M.R. \& Yang, R.J., A boundary element approach for recovery of shape sensitivities in three dimensional elastic solids. Comp. Meth. in Appl. Mech. Engng., 74, pp. 69-82, 1989.

[4] Matsumoto, T., Tanaka, M., Miyagawa, M. \& Ishii, N., Optimum design of cooling lines in injection moulds by using boundary element design sensitivity analysis. Finite Elements in Analysis and Design, 14, pp. 177-185, 1993.

[5] Matsumoto, T., Tanaka, M. \& Yamada, Y., Design Sensitivity Analysis of Steady-State Acoustic Problems using Boundary Integral Equation Formulation. JSME International Journal, Series C, 38(1), pp. 9-16, 1995.

[6] Haug, E.J., Choi, K.K. \& Komkov, V., Design Sensitivity Analysis of Structural Systems, Academic Press, 1986.

[7] Choi, J.H. \& Kwak, B.M., Boundary integral equation method for shape optimization of elastic structures, Int. J. Numer. Meth. Engng., 26, pp. 15791595, 1988.

[8] Brebbia, C.A., Telles, J.C.F. \& Wrobel, L.C., Boundary Element Techniques: Theory and Applications in Engineering, Springer, 1984,

[9] Arora, J.S., Lee, T.H. \& Cardoso, J.B., Structural shape sensitivity analysis: relationship between material derivative and control volume approach, AIAA J., 30(6), pp. 1638-1648, 1992. 